\title{
873.
}

\section{SYSTEM OF EQUATIONS FOR THREE CIRCLES WHICH CUT EACH OTHER AT GIVEN ANGLES.}

[From the Messenger of Mathematics, vol. xvII. (1888), pp. 18-21.]

Consider a triangle $A B C$, angles $A, B, C,(A+B+C=\pi)$ : to fix the absolute magnitude, assume that the radius of the circumscribed circle is $=1$; the lengths of the sides are therefore $=2 \sin A, 2 \sin B, 2 \sin C$ respectively. On the three sides as bases, outside of each, describe isosceles triangles $a B C, b C A, c A B$, the base angles whereof are $=\alpha, \beta, \gamma$ respectively. If we draw a circle touching $a B, a C$ at the points $B, C$ respectively; a circle touching $b C, b A$ at the points $C, A$ respectively; and a circle touching $c A, c B$ at the points $A, B$ respectively; then these circles form a curvilinear triangle $A B C$, the angles whereof are $A+\beta+\gamma, B+\gamma+\alpha, C+\alpha+\beta$ respectively. Taking as origin the centre of the circumscribed circle, and through this point, for axis of $x$, an arbitrary line, its position determined by the angle $\theta$, I write for conveniènce

$$
\begin{array}{lll}
F=\theta+2 B, & F^{\prime}=\theta-A, & A^{\prime}=A+\beta+\gamma, \\
G=\theta+2 B+2 C, & G^{\prime}=\theta+B, & B^{\prime}=B+\gamma+\alpha \\
H=\theta, & H^{\prime}=\theta+2 B+C, & C^{\prime}=C+\alpha+\beta
\end{array}
$$

then the coordinates of the angular points $A, B, C$ are $\left(\cos F^{\prime}, \sin F^{\prime}\right),(\cos G, \sin G)$, $(\cos H, \sin H)$ respectively; and the equations of the three circles are

$$
\begin{aligned}
& \left\{x+\frac{\sin (A-\alpha)}{\sin \alpha} \cos F^{\prime}\right\}^{2}+\left\{y+\frac{\sin (A-\alpha)}{\sin \alpha} \sin F^{\prime}\right\}^{2}=\frac{\sin ^{2} A}{\sin ^{2} \alpha} \\
& \left\{x+\frac{\sin (B-\beta)}{\sin \beta} \cos G^{\prime}\right\}^{2}+\left\{y+\frac{\sin (B-\beta)}{\sin \beta} \sin G^{\prime}\right\}^{2}=\frac{\sin ^{2} B}{\sin ^{2} \beta} \\
& \left\{x+\frac{\sin (C-\gamma)}{\sin \gamma} \cos H^{\prime}\right\}^{2}+\left\{y+\frac{\sin (C-\gamma)}{\sin \gamma} \sin H^{\prime}\right\}^{2}=\frac{\sin ^{2} C}{\sin ^{2} \gamma}
\end{aligned}
$$

respectively. 
In verification, observe that we have

$$
\begin{aligned}
& G-H=2 \pi-2 A, \quad G^{\prime}-H^{\prime}=-\pi+A, \quad G-F^{\prime}=2 \pi-A, \quad H-F^{\prime}=A, \\
& H-F=-2 B, \quad H^{\prime}-F^{\prime}=\pi+B, \quad H-G^{\prime}=-B, \quad F-G^{\prime}=B, \\
& F^{\prime}-G=-2 C, \quad F^{\prime}-G^{\prime}=-\pi+C, \quad F-H^{\prime}=-C^{\prime}, \quad G-H^{\prime}=C ;
\end{aligned}
$$

hence

$(\cos G-\cos H)^{2}+(\sin G-\sin H)^{2}=2-2 \cos (G-H), \quad=2(1-\cos 2 A), \quad=4 \sin ^{2} A$, and we thus see that the $\operatorname{sides}$ are $=2 \sin A, 2 \sin B, 2 \sin C$ respectively.

The first circle should pass through the points $(\cos G, \sin G),(\cos H, \sin H)$; we ought therefore to have, for the first of these points,

that is,

$$
1+2 \frac{\sin (A-\alpha)}{\sin \alpha} \cos (G-F)+\frac{\sin ^{2}(A-\alpha)}{\sin ^{2} \alpha}=\frac{\sin ^{2} A}{\sin ^{2} \alpha},
$$

$$
1+2 \frac{\sin (A-\alpha)}{\sin \alpha} \cos A+\frac{\sin ^{2}(A-\alpha)}{\sin ^{2} \alpha}=\frac{\sin ^{2} A}{\sin ^{2} \alpha}
$$

and, for the second of the points, the same equation. Write for a moment

$$
X=\frac{\sin A}{\sin \alpha}, \text { then } \frac{\sin (A-\alpha)}{\sin \alpha}=X \cos \alpha-\cos A ;
$$

then the equation is

that is,

$$
1+2(X \cos \alpha-\cos A) \cos A+(X \cos \alpha-\cos A)^{2}=X^{2},
$$

which is right.

$$
1-\cos ^{2} A=X^{2} \sin ^{2} \alpha,
$$

The second and third circles should intersect at the angle $A^{\prime}$, that is, we ought to have

$$
\begin{gathered}
\left\{\frac{\sin (B-\beta)}{\sin \beta} \cos G^{\prime}-\frac{\sin (C-\gamma)}{\sin \gamma} \cos H^{\prime}\right\}^{2}+\left\{\frac{\sin (B-\beta)}{\sin \beta} \sin G^{\prime}-\frac{\sin (C-\gamma)}{\sin \gamma} \sin H^{\prime}\right\} \\
=\frac{\sin ^{2} B}{\sin ^{2} \beta}+\frac{\sin ^{2} C}{\sin ^{2} \gamma}+2 \frac{\sin B \sin C}{\sin 3 \sin \gamma} \cos A^{\prime},
\end{gathered}
$$

or, reducing and for $\cos \left(G^{\prime}-H^{\prime}\right)$ substituting its value, $=-\cos A$, the equation is $\frac{\sin ^{2}(B-\beta)}{\sin ^{2} \beta}+\frac{\sin ^{2}(C-\gamma)}{\sin ^{2} \gamma}+2 \frac{\sin (B-\beta) \sin (C-\gamma)}{\sin \beta \sin \gamma} \cos A=\frac{\sin ^{2} B}{\sin ^{2} \beta}+\frac{\sin ^{2} C}{\sin ^{2} \gamma}+2 \frac{\sin B \sin C}{\sin \beta \sin \gamma} \cos A^{\prime}$.

Writing here

the equation is

$$
\frac{\sin B}{\sin \beta}=Y, \frac{\sin C}{\sin \gamma}=Z,
$$

$$
\begin{gathered}
(Y \cos \beta-\cos B)^{2}+(Z \cos \gamma-\cos C)^{2}+2(Y \cos \beta-\cos B)(Z \cos \gamma-\cos C) \cos A \\
=Y^{2}+Z^{2}+2 Y Z \cos A^{\prime},
\end{gathered}
$$


viz. this is

$$
Y^{2} \cos ^{2} \beta+Z^{2} \cos ^{2} \gamma+2 Y Z \cos \beta \cos \gamma \cos A
$$

$-2 Y \cos \beta(\cos B+\cos C \cos A)-2 Z \cos \gamma(\cos C+\cos A \cos B)$

$+\cos ^{2} B+\cos ^{2} C+2 \cos A \cos B \cos C=Y^{2}+Z^{2}+2 Y Z \cos A^{\prime}$.

Reducing by the relation $A+B+C=\pi$, this becomes

$-2 Y \cos \beta \sin A \sin C-2 Z \cos \gamma \sin A \sin B+1-\cos ^{2} A$

$$
=Y^{2} \sin ^{2} \beta+Z^{2} \sin ^{2} \gamma+2 Y Z\left(\cos A^{\prime}-\cos \beta \cos \gamma \cos A\right) .
$$

Here $A^{\prime}=A+\beta+\gamma$, and thence

$\cos A^{\prime}=\cos A(\cos \beta \cos \gamma-\sin \beta \sin \gamma)-\sin A(\sin \gamma \cos \beta+\sin \beta \cos \gamma) ;$

hence the right-hand is

$$
=Y^{2} \sin ^{2} \beta+Z^{2} \sin ^{2} \gamma-2 Y Z(\cos A \sin \beta \sin \gamma+\sin A \sin \gamma \cos \beta+\sin A \sin \beta \cos \gamma),
$$

or, reducing by

this is

$$
Y \sin \beta=\sin B, \quad Z \sin \gamma=\sin C,
$$

$=\sin ^{2} B+\sin ^{2} C-2 \sin B \sin C \cos A-2 Y \cos \beta \sin A \sin C-2 Z \cos \gamma \sin A \sin B$, and the terms in $Y, Z$ are equal to the like terms on the left-hand; the whole equation thus becomes

$$
-1+\cos ^{2} A+\sin ^{2} B+\sin ^{2} C-2 \cos A \sin B \sin C=0,
$$

where the last term is

$$
\begin{aligned}
& =2 \cos A\{\cos (B+C)-\cos B \cos C\} \\
& =-2 \cos ^{2} A-2 \cos A \cos B \cos C \\
& =-2 \cos ^{2} A+\left(\cos ^{2} A+\cos ^{2} B+\cos ^{2} C-1\right) \\
& =-\cos ^{2} A+\cos ^{2} B+\cos ^{2} C-1
\end{aligned}
$$

the equation is thus

$$
-1+\cos ^{2} A+\sin ^{2} B+\sin ^{2} C-\cos ^{2} A+\cos ^{2} B+\cos ^{2} C-1=0,
$$

or, finally, it is $-1+1+1-1=0$, which is an identity. The formulæ for the intersection of the third and first circles, and for that of the first and second circles, are of course precisely similar to the above formula for the intersection of the second and third circles; and the verifications are thus completed.

Cambridge, April 7, 1887.

C. XII. 